\title{
The boundary of a class of Rauzy fractals
}

\author{
Jefferson L. Bastos \\ Depto de Matemática, IBILCE, UNESP, \\ 15054-000, São José do Rio Preto, SP \\ E-mail: jeferson@ibilce.unesp.br \\ Tatiana Miguel Rodrigues \\ Depto de Matemática, FC, UNESP \\ 17033-360, Bauru, SP \\ E-mail: tatimi@fc.unesp.br.
}

Resumo: In this work we give arithmetical properties for the boundary of a class of Rauzy fractals $R_{a}$ given by the polynomial $x^{3}-a x^{2}+x-1, a \geq 3$. We give an automaton that generates this boundary and we prove that it is homeomorphic to $S^{1}$.

Palavras-chave: Rauzy fractal, Tiling, Automaton

\section{Introduction}

The Rauzy fractal was studied by many mathematicians and was connected to to many topics as: numeration systems $([8],[6])$, geometrical representation of symbolic dynamical system ([2], [7]), multidimensional continued fractions and simultaneous approximations ([3], [5]), auto-similar tilings ([2], [8]) and Markov partitions of Hyperbolic automorphisms of Torus ([7], [8]). There are many ways of constructing Rauzy's fractals one of them is by $\beta$-expansions.

Let $\beta>1$ be a fixed real number and $x$ any positive real number. Using Greedy algorithm we can write $x=\sum_{i=N_{0}}^{\infty} a_{-i} \beta^{-i}, a_{-i} \in \mathbb{Z} \cap[0, \beta)(\beta$ expansion of $x)$. A Pisot number $\beta>1$ is an algebraic integer whose conjugates other than itself have modulus less than one. Let Fin $(\beta)$ be a set consisting of all finite $\beta$-expansion and consider the condition

$$
\operatorname{Fin}(\beta)=\mathbb{Z}\left[\beta^{-1}\right] \cap \mathbb{R}^{+} \quad(\text { property } F)
$$

The Pisot numbers that satisfy property $(\mathrm{F})$ were characterized in [1] as being exactly the set of dominant roots of the polynomial (with integers coefficients)

$$
P_{a, b}(x)=x^{3}-a x^{2}-b x-1, a \geq 0,-1 \leq b \leq a+1 .
$$

(If $b=-1$ add the restriction $a \geq 2$ ). In particular, this set is divided into three subsets:

a) $0 \geq b \geq a$, and in this case $d(1, \beta)=\cdot a b 1$.

b) $b=-1, a \geq 2$. In this case $d(1, \beta)=\cdot(a-1)(a-1) 01$.

c) $b=a+1$, and in this case $d(1, \beta)=\cdot(a+1) 00 a 1$, where $d(1, \beta)$ is the Rényi $\beta$-representation of 1 (see citerényi).

We can associated a fractal to each of this cases above. The fractal associated to $(b)$ is given by

$$
\mathcal{R}_{a}=\left\{\sum_{i=2}^{\infty} a_{i} \alpha^{i}, a_{i} a_{i-1} a_{i-2} a_{i-3}<_{l e x}(a-1)(a-1) 01, \forall i \geq 5\right\},
$$


where $<_{\text {lex }}$ is the lexicographic order on finite words. In [4] we prove some topological and arithmetic properties of $\mathcal{R}_{a}$ and give a complete description of the boundary of $\mathcal{R}_{2}$. The purpose of this work is to present a complete description of the boundary of $\mathcal{R}_{a}, a \geq 3$. For this we need the following results:

Theorem 1.1. $R_{a}$ induces a periodic tiling of the plane $\mathbb{C}$ modulo $\mathbb{Z}(\alpha-1)+\mathbb{Z}\left(\alpha^{2}-\alpha\right)$. Moreover

$$
\partial R_{a}=\bigcup_{v \in B}\left(R_{a} \cap\left(R_{a}+v\right)\right), B=\left\{ \pm(\alpha-1) ; \pm\left(\alpha^{2}-\alpha\right) ; \pm\left(\alpha^{2}-1\right) ; \pm\left(\alpha^{2}-2 \alpha+1\right)\right\}
$$

$R_{a} \cap\left(R_{a}+\left(\alpha^{2}-1\right)\right)=\{-1\} ; R_{a} \cap\left(R_{a}+\left(\alpha^{2}-2 \alpha+1\right)\right)=\{-\alpha\}$.

Theorem 1.2. Consider $g(z)=\alpha-1+\alpha(z)$. Then

$$
R_{\alpha-1}=R_{a} \cap\left(R_{a}+\alpha-1\right)=g\left(R_{a} \cap\left(R_{a}+\left(\alpha^{2}-\alpha\right)\right)\right)
$$

\section{Parametrization of the boundary of $\mathcal{R}_{a}, \forall a \geq 3$}

In this section we give a complete description of the boundary of $R_{a}$. By theorem 1.1 we have to study the sets $R_{a} \cap\left(R_{a}+v\right), v \in\left\{ \pm(\alpha-1), \pm\left(\alpha^{2}-\alpha\right)\right\}$. By symmetry and theorem 1.2 we can just study the set $R_{\alpha-1}$.

In [4] we show that the automaton below characterize the boundary of $\mathcal{R}_{a}$.

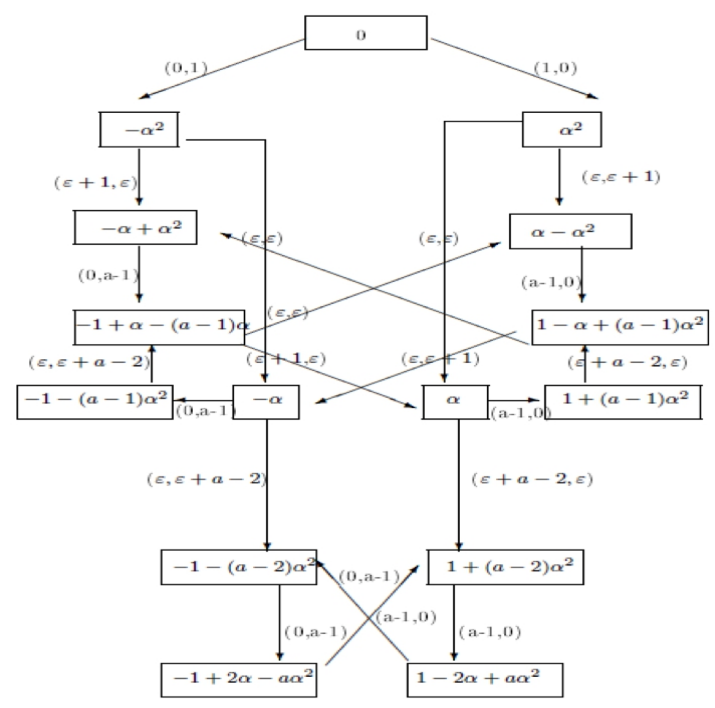

Figure 1: Automaton $\mathcal{A}$

Using the automaton we have $R_{\alpha-1}=R_{\alpha-1}^{1} \cup R_{\alpha-1}^{2}$ where $R_{\alpha-1}^{1}=\left\{z=\alpha-1+\sum_{i=3} a_{i} \alpha^{i}=(a-1) \alpha^{2}+\sum_{i=3} b_{i} \alpha^{i},\left(a_{3}, b_{3}\right)=(\epsilon, \epsilon), \epsilon=0, \ldots, a-1\right\}$, $R_{\alpha-1}^{2}=\left\{z=\alpha-1+\sum_{i=3} a_{i} \alpha^{i}=(a-1) \alpha^{2}+\sum_{i=3} b_{i} \alpha^{i},\left(a_{3}, b_{3}\right)=(\epsilon+1, \epsilon), \epsilon=0, \ldots, a-2\right\}$.

Lemma 2.1. Considering $R_{\alpha-1}^{1, t}=\left\{z \in R_{\alpha-1}^{1} ;\left(a_{3}, b_{3}\right)=(t, t), t=0,1 \ldots a-1\right\}$, $R_{\alpha-1}^{2, t}=\left\{z \in R_{\alpha-1}^{2} ;\left(a_{3}, b_{3}\right)=(t, t-1), t=1,2 \ldots a-1\right\}$ and $R_{\alpha-1}^{\prime}=\left\{z \in R_{\alpha-1} ; a_{3} \neq a-1\right\}$. We have:

1. $g_{2 k+1}: R_{\alpha-1} \longrightarrow R_{\alpha-1}^{2, a-1-k}$ given by $g_{2 k+1}(z)=-1-k \alpha^{3}+\alpha^{3} z, k=0, \ldots, a-2$ is bijective.

2. $g_{2(a-1)}: R_{\alpha-1} \longrightarrow R_{\alpha-1}^{1,0}$ given by $g_{2(a-1)}(z)=\alpha-1+\alpha^{2} z$ is bijective.

3. $g_{2 k}: R_{\alpha-1}^{\prime} \longrightarrow R_{\alpha-1}^{1, a-1-k}$ given by $g_{2 k}(z)=\alpha-1+(a-1-k) \alpha^{3}+\alpha^{2} z, k=0, \ldots, a-2$ is bijective. 


\section{Corollary 2.2.}

$$
R_{\alpha-1}=\bigcup_{i=0}^{2(a-1)} g_{i}(X)
$$

where $X=R_{\alpha-1}$ if $i$ is odd and $2(a-1)$ and $X=R_{\alpha-1}^{\prime}$ if $i$ is even.

Using the previous notation and taking $u=-1, v=-(a-1) \alpha-\alpha^{-1}, w=-1-\alpha^{3}$ we have the following lemma.

Lemma 2.3. $g_{2 k}\left(-1-\alpha^{3}\right)=-1-\alpha^{2}-k \alpha^{3}-(a-1) \alpha^{4}=g_{2 k+1}\left(-(a-1) \alpha-\alpha^{-1}\right), k=0, \ldots, a-2$, and $g_{2 k+1}(-1)=-1-(k+1) \alpha^{3}=g_{2(k+1)}\left(-(a-1) \alpha-\alpha^{-1}\right), k=0, \ldots, a-2$.

Lemma 2.4. Take $r=2 a-1$. Then we have

1. $\lim _{n \longrightarrow \infty}\left(g_{0} \circ g_{r-1}\right)^{n}(z)=u=-1$.

2. $\lim _{n \rightarrow \infty}\left(g_{r-1} \circ g_{0}\right)^{n}(z)=v=-(a-1) \alpha-\alpha^{-1}, \forall z \in R_{\alpha-1}^{\prime}$.

Lemma 2.5. Take $t \in[0,1], a \geq 3, r=2 a-1$. Then

1. $t=\frac{a_{1}}{r}+B+\sum_{\substack{k=3 \\ m_{k}+n_{k}=k}}^{\infty} \frac{a_{k}}{(r-2)^{m_{k}} r^{n_{k}}}$ where

(a) $B= \begin{cases}\frac{a_{2}}{r^{2}} & , a_{2} \in\{0,1,2, \ldots, r-1\} \text { if } a_{1} \in\{1,3,5, \ldots, r-2, r-1\} \\ \frac{a_{2}}{(r-2) r} & , \quad a_{2} \in\left\{0^{*}, 1^{*}, \ldots,(r-3)^{*}\right\} \text { if } a_{1} \in\{0,2,4, \ldots, r-3\}\end{cases}$

and for $i \geq 3$ we have:

(b) If $a_{i-1}=0,0^{*}, i-1$ even or $a_{i-1}=(r-3)^{*}, r-1, i-1$ odd or $a_{i-1}=r-2,(2 n-$ $1),(2 n-1)^{*}, n=1, \ldots, a-2$ then $a_{i} \in\{0,1,2 \ldots, r-1\}, m_{i}=m_{i-1}$ and $n_{i}=n_{i-1}+1$.

(c) If $a_{i-1}=0,0^{*}, i-1$ odd or $a_{i-1}=(r-3)^{*}, r-1, i-1$ even or $a_{i-1}=r-$ $3,(2 n),(2 n)^{*}, n=1, \ldots, a-3$ then $a_{i} \in\left\{0^{*}, 1^{*}, \ldots(r-3)^{*}\right\}, m_{i}=m_{i-1}+1$ and $n_{i}=n_{i-1}$.

2. If $\left|t^{\prime}-t\right|<(r-2)^{m} r^{n}$ with $m+n=N$ then there exists $k<N$ such that

(a) $t=a_{1} \ldots a_{k-1} a_{k}(r-1)(r-1)(r-3)^{*}(r-1)(r-3)^{*} \ldots a_{N+1} \ldots, t^{\prime}=a_{1} \ldots a_{k-1}\left(a_{k}+\right.$ 1) $00 \ldots a_{N+1}^{\prime} \ldots$ if $k$ is even and $a_{k}=0,0^{\star}, r-2,(2 n-1),(2 n-1)^{*}, n=1, \ldots, a-2$.

(b) $t=a_{1} \ldots a_{k-1} a_{k}(r-1)(r-3)^{*}(r-1)(r-3)^{*} \ldots a_{N+1} \ldots, t^{\prime}=a_{1} \ldots a_{k-1}\left(a_{k}+1\right) 00 \ldots a_{N+1}^{\prime} \ldots$ if $k$ is odd and $a_{k}=r-2,(2 n-1),(2 n-1)^{*}, n=1, \ldots, a-2$.

(c) $t=a_{1} \ldots a_{k-1} a_{k}(r-3)^{*}(r-3)^{*}(r-1)(r-3)^{*}(r-1) \ldots a_{N+1} \ldots, t^{\prime}=a_{1} \ldots a_{k-1}\left(a_{k}+\right.$ 1) $00 \ldots a_{N+1}^{\prime} \ldots$ if $k$ is odd and $a_{k}=0,0^{\star}, r-3,(2 n),(2 n)^{*}, n=1, \ldots, a-3$.

(d) $t=a_{1} \ldots a_{k-1} a_{k}(r-3)^{*}(r-1)(r-3)^{*}(r-1) \ldots a_{N+1} \ldots, t^{\prime}=a_{1} \ldots a_{k-1}\left(a_{k}+1\right) 00 \ldots a_{N+1}^{\prime} \ldots$ if $k$ is even and $a_{k}=r-3,(2 n),(2 n)^{*}, n=1, \ldots, a-3$.

\section{Proof:}

1. Take $t, t^{\prime} \in[0,1],\left|t^{\prime}-t\right|<(r-2)^{m} r^{n}$ with $m+n=N$ and suppose $t<t^{\prime}$. Then exist $k \in$ $\mathbb{N}, k<N$ such that $t=a_{1} \ldots a_{k-1} a_{k} a_{k+1} \ldots a_{N} a_{N+1} \ldots, t^{\prime}=a_{1} \ldots a_{k-1} a_{k}^{\prime} a_{k+1}^{\prime} \ldots a_{N}^{\prime} a_{N+1}^{\prime} \ldots, a_{k}<$ $a_{k}^{\prime}$ and

$$
t^{\prime}-t=\frac{\left(a_{k}^{\prime}-a_{k}\right)}{(r-2)^{m_{k}} r^{n_{k}}}+\ldots=\frac{\left(a_{k}^{\prime}-a_{k}-1\right)}{(r-2)^{m_{k}} r^{n_{k}}}+\frac{1}{(r-2)^{m_{k}} r^{n_{k}}}+\ldots
$$

As $m_{k}+n_{k}=k$ and $\left|t^{\prime}-t\right|<(r-2)^{m} r^{n}, m+n>N>k$ then $a_{k}^{\prime}-a_{k}-1=0$, that is, $a_{k}^{\prime}=a_{k}+1$. 
(a) Let $k$ be an even number and $a_{k}=0,0^{*}, r-2,(2 n-1),(2 n-1)^{*}, n=1, \ldots, a-2$. Then $a_{k+1} \in\{0,1, \ldots, r-1\}$ and we can write

$$
\frac{1}{(r-2)^{m_{k}} r^{n_{k}}}=\frac{r-1}{(r-2)^{m_{k}} r^{n_{k}+1}}+\sum_{i=0}^{\infty}\left(\frac{r-1}{(r-2)^{m_{k}+i} r^{n_{k}+2+i}}+\frac{(r-3)^{*}}{(r-2)^{m_{k}+1+i} r^{n_{k}+2+i}}\right) .
$$

Therefore

$$
\begin{aligned}
t^{\prime}-t & =\frac{a_{k+1}^{\prime}}{(r-2)^{m_{k+1}^{\prime}} r^{n_{k+1}^{\prime}}}-\frac{a_{k+1}}{(r-2)^{m_{k}} r^{n_{k}+1}}+\frac{(r-1)}{(r-2)^{m_{k}} r^{n_{k}+1}}+\ldots= \\
& =\frac{a_{k+1}^{\prime}}{(r-2)^{m_{k+1}^{\prime}} r^{n_{k+1}^{\prime}}}+\frac{r-1-a_{k+1}}{(r-2)^{m_{k}} r^{n_{k}+1}}+\ldots
\end{aligned}
$$

where $m_{k+1}^{\prime}+n_{k+1}^{\prime}=m_{k}+n_{k}+1=k+1$. As $\left|t^{\prime}-t\right|<(r-2)^{m} r^{n}, m+n>N \geq k+1$ then $\frac{a_{k+1}^{\prime}}{(r-2)^{m_{k+1}^{\prime} r^{n_{k+1}^{\prime}}}}+\frac{r-1-a_{k+1}}{(r-2)^{m} k r^{n} k^{+1}}=0$ and it is possible only with $a_{k+1}^{\prime}=0,0^{*}$ and $a_{k+1}=r-1$.

As $a_{k+1}=r-1$ and $k+1$ is an odd number, then $a_{k+2} \in\{0,1, \ldots, r-1\}$ we have

$$
\begin{aligned}
t^{\prime}-t & =\frac{a_{k+2}^{\prime}}{(r-2)^{m_{k+2}^{\prime}} r^{n_{k+2}^{\prime}}}-\frac{a_{k+2}}{(r-2)^{m_{k}} r^{n_{k}+2}}+\frac{r-1}{(r-2)^{m_{k}} r^{n_{k}+2}}+\ldots= \\
& =\frac{a_{k+2}^{\prime}}{(r-2)^{m_{k+2}^{\prime}} r^{n_{k+2}^{\prime}}}+\frac{r-1-a_{k+2}}{(r-2)^{m_{k}} r^{n_{k}+2}}+\ldots
\end{aligned}
$$

with $m_{k+2}^{\prime}+n_{k+2}^{\prime}=m_{k}+n_{k}+2=k+2$. Again we have $a_{k+2}^{\prime}=0,0^{*}$ e $a_{k+2}=r-1$. Now $a_{k+2}=r-1$ and $k+2$ is an even number. Then $a_{k+3} \in\left\{0^{*}, 1^{*}, \ldots(r-3)^{*}\right\}$ and

$$
\begin{aligned}
t^{\prime}-t & =\frac{a_{k+3}^{\prime}}{(r-2)^{m_{k+3}^{\prime}} r^{n_{k+3}^{\prime}}}-\frac{a_{k+3}}{(r-2)^{m_{k}+1} r^{n_{k}+2}}+\frac{(r-3)^{*}}{(r-2)^{m_{k}+1} r^{n_{k}+2}}+\ldots= \\
& =\frac{a_{k+3}^{\prime}}{(r-2)^{m_{k+3}^{\prime}} r^{n_{k+3}^{\prime}}}+\frac{(r-3)^{*}-a_{k+3}}{(r-2)^{m_{k}+1} r^{n_{k}+2}}+\ldots
\end{aligned}
$$

with $m_{k+3}^{\prime}+n_{k+3}^{\prime}=m_{k}+n_{k}+3=k+3$. Therefore $a_{k+3}^{\prime}=0,0^{*}$ e $a_{k+3}=(r-3)^{*}$. Following this idea we have the result.

Following this idea we can prove the others items.

Corollary 2.6. Using the notations of lemma 2.5, if $t, t^{\prime} \in[0,1]$ then $t=t^{\prime}$ if and only if

1. $t=a_{1} \ldots a_{k-1} a_{k}(r-1) \overline{(r-1)(r-3)^{*}}, t^{\prime}=a_{1} \ldots a_{k-1}\left(a_{k}+1\right) \overline{0}$ or;

2. $t=a_{1} \ldots a_{k-1} a_{k} \overline{(r-1)(r-3)^{*}}, t^{\prime}=a_{1} \ldots a_{k-1}\left(a_{k}+1\right) \overline{0}$ or ;

3. $t=a_{1} \ldots a_{k-1} a_{k}(r-3)^{*} \overline{(r-3) *(r-1)}, t^{\prime}=a_{1} \ldots a_{k-1}\left(a_{k}+1\right) \overline{0}$ or;

4. $t=a_{1} \ldots a_{k-1} a_{k} \overline{(r-3)^{*}(r-1)}, t^{\prime}=a_{1} \ldots a_{k-1}\left(a_{k}+1\right) \overline{0}$.

Let $A=\{0,1, \ldots, r-1\}$ be a subset of $\mathbb{N}$ and consider

$$
\begin{aligned}
\psi: \quad & A^{\mathbb{N}} \longrightarrow A^{\mathbb{N}} \\
& \left(a_{i}\right) \longmapsto\left(b_{i}\right)
\end{aligned}
$$


given by

$b_{1}=a_{1}$;

$b_{2 k}=r-1-a_{2 k}$;

$b_{2 k+1}=a_{2 k+1}$ if $a_{2 k} \in\left\{0,0^{*}, 2 n-1,(2 n-1)^{*}, n=1 \ldots, a-2, r-2\right\}$;

$b_{2 k+1}=a_{2 k+1}+2$ if $a_{2 k} \in\left\{2 n,(2 n)^{*}, n=1, \ldots, a-2, r-1\right\}$.

Take $x_{0} \in R_{\alpha-1}^{\prime}$ and consider $f:[0,1] \longrightarrow R_{\alpha-1}$ defined as follows:

if $t=\sum_{i=1}^{\infty} a_{i}(r-2)^{-m_{i}} r^{-n_{i}},\left(a_{i}\right) \in A^{\mathbb{N}}$, then $f(t)=\lim _{n \rightarrow \infty} g_{b_{1}} \circ g_{b_{2}} \circ \ldots \circ g_{b_{n}}\left(x_{0}\right)$ where $\psi\left(a_{1} a_{2} \ldots\right)=$ $b_{1} b_{2} \ldots$

Theorem 2.7. $f$ is a continue and bijective function satisfying $f(0)=u$ and $f(1)=v$.

\section{Proof:}

1. $f$ is a well defined function.

We are going to use the following notation: $g_{b_{1}} \ldots g_{b_{k-1}} g_{b_{k}}(z)=b_{1} \ldots b_{k}$.

According lemma 2.4 we have

$$
\begin{aligned}
& u=-1=0(r-1) 0(r-1) \ldots=\overline{0(r-1)} . \\
& v=-(a-1) \alpha-\alpha^{-1}=(r-1) 0(r-1) 0 \ldots=\overline{(r-1) 0} \\
& w=-1-\alpha^{3}=2(r-1) 0(r-1) 0 \ldots=2 \overline{(r-1) 0} .
\end{aligned}
$$

We have to consider all the cases of corollary 2.6 .

(a) Let $k$ be an even number and $a_{k}=0,0^{*}, r-2,(2 n-1),(2 n-1)^{*}, n=1, \ldots, a-2, a_{k}+1=$ $1,1^{*}, r-1,2 n,(2 n)^{*}$.

Suppose that $t=a_{1} \ldots a_{k-1} a_{k}(r-1) \overline{(r-1)(r-3)^{*}}=a_{1} \ldots a_{k-1}\left(a_{k}+1\right) \overline{0}=t^{\prime}$. Then: $f(t)=b_{1} \ldots b_{k-1}(r-1) \overline{(r-1) 0}$ and $f\left(t^{\prime}\right)=b_{1} \ldots b_{k-1}(r-2) \overline{0(r-1)}$, if $a_{k}=0,0^{*}$;

$f(t)=b_{1} \ldots b_{k-1}(r-2 n) \overline{(r-1) 0}$ and $f\left(t^{\prime}\right)=b_{1} \ldots b_{k-1}(r-2 n-1) 2 \overline{(r-1) 0}$ if $a_{k}=2 n-1,(2 n-1)^{*}$;

$f(t)=b_{1} \ldots b_{k-1} 1 \overline{(r-1) 0}$ and $f\left(t^{\prime}\right)=b_{1} \ldots b_{k-1} 02 \overline{(r-1) 0}$ if $a_{k}=r-2$.

For all these cases we have $f(t)=f\left(t^{\prime}\right)$.

(b) Let $k$ be an odd number and $a_{k}=r-2,(2 n-1),(2 n-1)^{*}, n=1, \ldots, a-2$.

(c) Let $k$ be an odd number and $a_{k}=0,0^{*}, r-3,(2 n),(2 n)^{*}, n=1, \ldots, a-3$.

(d) Let $k$ be an even number and $a_{k}=r-3,(2 n),(2 n)^{*}, n=1, \ldots, a-3$.

For all these cases the proof is similar to (a).

2. Suppose that $f(t)=f\left(t^{\prime}\right)$. According to lemma 2.3 we have two possibilities:

(a) $f(t)=g_{b_{1}} \ldots g_{b_{k-1}} g_{b_{k}}(-1), b_{k} \in\{1,3,5, \ldots, r-2\}$ and $f\left(t^{\prime}\right)=g_{b_{1}} \ldots g_{b_{k-1}} g_{b_{k}+1}(v)$.

Using the above notations we have

$f(t)=b_{1} \ldots b_{k-1} b_{k} \overline{0(r-1)}$ and $f\left(t^{\prime}\right)=b_{1} \ldots b_{k-1}\left(b_{k}+1\right) \overline{(r-1) 0}$.

i. $k$ is an even number, $b_{k} \neq r-2$.

In this case $b_{k}=r-1-a_{k}$ and then $a_{k}=r-1-b_{k}$ is an odd number. According to the rules:

$-b_{k+1}=0, a_{k}$ odd number $\Rightarrow a_{k+1}=0$.

- $b_{k+2}=r-1, k+2$ even number $\Rightarrow a_{k+2}=0$.

- $b_{k+3}=0, a_{k+2}=0 \Rightarrow a_{k+3}=0$.

Therefore $t=a_{1} \ldots a_{k-1}\left(r-1-b_{k}\right) \overline{0}$.

We also have $b_{k}^{\prime}=b_{k}+1=r-1-a_{k}^{\prime}$ and then $a_{k}^{\prime}=r-2-b_{k} \neq 0$ is an even number. According to the rules:

- $b_{k+1}^{\prime}=r-1, a_{k}^{\prime}$ even $\Rightarrow a_{k+1}^{\prime}=(r-3)^{*}$.

- $b_{k+2}^{\prime}=0, k+2$ even $\Rightarrow a_{k+2}^{\prime}=r-1$.

- $b_{k+3}^{\prime}=r-1, a_{k+2}^{\prime}=r-1 \Rightarrow a_{k+3}=(r-3)^{*}$.

Therefore $t^{\prime}=a_{1} \ldots a_{k-1}\left(r-2-b_{k}\right) \overline{(r-3)^{*}(r-1)}$ and then $t=t^{\prime}$. 
ii. $k$ is an even number, $b_{k}=r-2$. In this case $b_{k}=r-1-a_{k}$ and then $a_{k}=$ $r-1-b_{k}=1$.

iii. $k$ is an odd number. In this case $b_{k}=a_{k}$ or $b_{k}=a_{k}+2$ and then $a_{k}=b_{k}$ or $a_{k}=b_{k}-2$. The proof is similar to $(i)$

(b) $f(t)=g_{b_{1}} \ldots g_{b_{k-1}} g_{b_{k}}\left(-1-\alpha^{3}\right), b_{k} \in\{0,2, \ldots, r-3\}$ and $f\left(t^{\prime}\right)=g_{b_{1} \ldots} g_{b_{k-1}} g_{b_{k}+1}(v)$. Here the proof is similar to (a).

3. $f$ is a continuous function

Let us consider $t, t^{\prime} \in[0,1]$ given by

$$
t=\frac{a_{1}}{r}+B+\sum_{\substack{k=3 \\ m_{k}+n_{k}=k}}^{\infty} \frac{a_{k}}{(r-2)^{m_{k}} r^{n_{k}}}, t^{\prime}=\frac{a_{1}^{\prime}}{r}+B^{\prime}+\sum_{\substack{k=3 \\ m_{k}^{\prime}+n_{k}^{\prime}=k}}^{\infty} \frac{a_{k}^{\prime}}{(r-2)^{m_{k}^{\prime}} r^{n_{k}^{\prime}}} .
$$

If $\left|t^{\prime}-t\right|<(r-2)^{m} r^{n}$ then according to lemma (2.5) we have to consider the following cases:

- $t=a_{1} \ldots a_{k-1} a_{k}(r-1)(r-1)(r-3)^{*}(r-1)(r-3)^{*} \ldots a_{N+1} \ldots, t^{\prime}=a_{1} \ldots a_{k-1}\left(a_{k}+\right.$ 1) $00 \ldots a_{N+1}^{\prime} \ldots$ if $k$ is even and $a_{k}=0,0^{\star}, r-2,(2 n-1),(2 n-1)^{*}, n=1, \ldots, a-2$. Using what was done before we have to consider the following:

(a) $f(t)=b_{1} \ldots b_{k-1}(r-1)(r-1) 0(r-1) 0 \ldots b_{N+1} \ldots$ and $f\left(t^{\prime}\right)=b_{1} \ldots b_{k-1}(r-2) 0(r-1) 0(r-$ 1)... $b_{N+1}^{\prime} \ldots, a_{k}=0,0^{*}$. Then

$$
\begin{aligned}
\left|f(t)-f\left(t^{\prime}\right)\right| & =\left|g_{b_{1}} \circ g_{b_{2}} \circ \ldots \circ g_{b_{k-1}} \circ g_{r-1}\left(z_{1}\right)-g_{b_{1}} \circ g_{b_{2}} \circ \ldots \circ g_{b_{k-1}} \circ g_{r-2}\left(z_{2}\right)\right| \leq \\
& \leq|\alpha|^{2(k-1)}\left|g_{r-1}\left(z_{1}\right)-g_{r-2}\left(z_{2}\right)\right| .
\end{aligned}
$$

As $g_{r-2}(u)=g_{r-1}(v)$ then

$$
\begin{aligned}
\left|f(t)-f\left(t^{\prime}\right)\right| & \leq|\alpha|^{2(k-1)}\left(\left|g_{r-1}\left(z_{1}\right)-g_{r-1}(v)\right|+\left|g_{r-2}\left(z_{2}\right)-g_{r-2}(u)\right|\right) \leq \\
& \leq|\alpha|^{2(k-1)}\left(|\alpha|^{2}+|\alpha|^{3}\right) \operatorname{diam}\left(R_{\alpha-1}\right)=|\alpha|^{2 k}(1+|\alpha|) \operatorname{diam}\left(R_{\alpha-1}\right) .
\end{aligned}
$$

(b) $f(t)=b_{1} \ldots b_{k-1}(r-2 n)(r-1) 0(r-1) 0 \ldots b_{N+1} \ldots$ and $f\left(t^{\prime}\right)=b_{1} \ldots b_{k-1}(r-2 n-1) 2(r-$ 1) $0(r-1) 0 \ldots b_{N+1}^{\prime} \ldots, a_{k}=2 n-1,(2 n-1)^{*}, n=1, \ldots, a-2$.

(c) $f(t)=b_{1} \ldots b_{k-1} 1(r-1) 0(r-1) 0 \ldots b_{N+1} \ldots$ and $f\left(t^{\prime}\right)=b_{1} \ldots b_{k-1} 02(r-1) 0(r-1) 0 \ldots b_{N+1}^{\prime} \ldots$, $a_{k}=r-2$.

- $t=a_{1} \ldots a_{k-1} a_{k}(r-1)(r-3)^{*}(r-1)(r-3)^{*} \ldots a_{N+1} \ldots, t^{\prime}=a_{1} \ldots a_{k-1}\left(a_{k}+1\right) 00 \ldots a_{N+1}^{\prime} \ldots$ if $k$ is odd and $a_{k}=r-2,(2 n-1),(2 n-1)^{*}, n=1, \ldots, a-2$.

- $t=a_{1} \ldots a_{k-1} a_{k}(r-3)^{*}(r-3)^{*}(r-1)(r-3)^{*}(r-1) \ldots a_{N+1} \ldots, t^{\prime}=a_{1} \ldots a_{k-1}\left(a_{k}+\right.$ 1) $00 \ldots a_{N+1}^{\prime} \ldots$ if $k$ is odd and $a_{k}=0,0^{\star}, r-3,(2 n),(2 n)^{*}, n=1, \ldots, a-3$.

- $t=a_{1} \ldots a_{k-1} a_{k}(r-3)^{*}(r-1)(r-3)^{*}(r-1) \ldots a_{N+1} \ldots, t^{\prime}=a_{1} \ldots a_{k-1}\left(a_{k}+1\right) 00 \ldots a_{N+1}^{\prime} \ldots$ if $k$ is even and $a_{k}=r-3,(2 n),(2 n)^{*}, n=1, \ldots, a-3$.

For these item the proof is similar. In all that cases we conclude that $f$ is a continuous function.

Theorem 2.8. The boundary of $\mathcal{R}_{a}, \forall a>2$ is homeomorphic to $S^{1}$.

\section{References}

[1] S. Akiyama, Cubic Pisot units with finite beta expansions, Algebraic Number Theory and Diophantine Analysis, ed. F. Halter-Koch and R.F. Tichy (2000) 11-26.

[2] P. Arnoux, S. Ito, Pisot substitutions and Rauzy fractals, Bull Belg. Math. Soc. Simon Stevin, 8 (2001) 181-207.

[3] P. Arnoux, V. Berthé, S. Ito, Discrete planes $\mathbb{Z}^{2}$-actions, Jacobi-Perron algorithm and substitutions, Ann. Inst. Fourier, 52 (2002) 305-349. 
[4] J. Bastos, A. Messaoudi, D. Smania, T. Rodrigues, A class of cubic Rauzy fractals, preprint.

[5] N. Chekhova, P. Hubert, A. Messaoudi, Propriétés combinatoires, ergodiques et arithmétiques de la substitution de Tribonacci, J. Theor. Nombres Bordeaux, 13 (2001) 371-394.

[6] A. Messaoudi, Frontière du fractal de Rauzy et systémes de numération complexe, Acta Arithmetica, XCV (2000) 195-224

[7] A. Messaoudi, Propriétés arithmétiques et dynamiques du fractal de Rauzy, J. Theor. Nombres Bordeaux, 10 (1998) 135-162.

[8] B. Praggastis, Numerations systems and Markov partitions from self similar tilings, Trans. Amer. Math. Soc., 351 (1999) 3315-3349. 